\title{
The risk and survival outcome of subsequent primary colorectal cancer after the first primary colorectal cancer: cases from 1973 to 2012
}

Jiao Yang ${ }^{1}$, Xianglin L. Du², Shuting $\mathrm{Li}^{1}$, Yinying $\mathrm{Wu}^{1}$, Meng Lv , Danfeng Dong ${ }^{1}$, Lingxiao Zhang ${ }^{1}$, Zheling Chen ${ }^{1}$, Biyuan Wang ${ }^{1}$, Fan Wang ${ }^{1}$, Yanwei Shen ${ }^{1}$, Enxiao $\mathrm{Li}^{1}, \mathrm{Min} \mathrm{Yi}^{3}$ and Jin Yang ${ }^{{ }^{*}}$

\begin{abstract}
Background: Among colorectal cancer (CRC) survivors, how the prior tumor location affects the risk of subsequent primary colorectal cancer (SPCRC) and the outcome of those suffering from SPCRC remain unknown.

Methods: CRC cases diagnosed from 1973 to 2012 were screened for SPCRC development using the Surveillance, Epidemiology, and End Results database. The relative risk of SPCRC was estimated using the standardized incidence ratio. Survivals were analyzed using the Kaplan-Meier and Cox regression model.

Results: The overall risk of SPCRC increased by $27 \%$ in CRC survivors compared to that of the general population. The risk increased in patients with both prior right colon cancer (RCC) and left colon cancer (LCC), and was concentrated in the first 5 years after the prior diagnosis, and among young patients. Among the 6701 SPCRC patients identified, patients with prior RCC were more likely to be elderly, female, and with more low or undifferentiated disease than those with prior LCC or rectal cancer (ReC). The overall survivals differed by both prior tumor location $(P<0.0001)$ and age $(P<0.0001)$, and the difference by tumor location remained significant when adjusted or stratified by any other potential prognostic factor except age. The cancer specific survivals differed by age $(P<0.0001)$ rather than by prior tumor location $(P=0.455)$.

Conclusions: The overall risk of SPCRC increased among patients with both prior RCC and LCC, but not among those with ReC. The different survival outcomes in CRC survivors suffering from SPCRC were largely explained by the patient age but not by the prior tumor location.
\end{abstract}

Keywords: Subsequent primary colorectal cancer, Tumor location, Age

\section{Background}

Colorectal cancer (CRC) is the third most common cancer worldwide and the second most common cause of cancerrelated deaths in Western countries. The survival of CRC patients has improved gradually with the widespread use of advanced diagnostic and therapeutic methods, including fecal occult bleeding tests, colonoscopy screening, targeted treatments, and multidisciplinary team therapy approaches.

\footnotetext{
* Correspondence: yangjin@mail.xjtu.edu.cn

${ }^{1}$ Departments of Medical Oncology, The First Affiliated Hospital of Xi'an Jiaotong University, 277 Yanta West Road, Xi'an 710061, Shaanxi Province, People's Republic of China

Full list of author information is available at the end of the article
}

The increased survival rate has resulted in increased risks of developing subsequent primary malignancies, among which the subsequent primary colorectal cancer (SPCRC) was the most commonly observed form of cancer $[1,2]$. Synchronous colorectal adenoma and family history of CRC are indicators for SPCRC [3-5]. Furthermore, the risk of SPCRC was reported to change over time and was influenced by other factors, including latency and age [6-8].

Tumors derived from different colorectal segments have distinct clinicopathological features and genetic variations. The risk of SPCRC was shown to differ by tumor location prior to CRC, but the studies remain controversial. Some studies indicated a high risk in patients 
with prior cancer located in the proximal colon $[9,10]$. Gervaz et al. [9] proposed that the proximal location of colon cancer was a risk factor for SPCRC based on a higher prevalence of SPCRC in patients with prior right colon cancer (RCC; 3.4\%) than those with prior left colon cancer (LCC), and rectal cancer ( $\mathrm{ReC} ; 1.8 \%)$ after longterm follow-up. However, other studies suggested a high risk in those patients with prior cancer located in the distal colon or rectum [11-13]. For example, Borda et al. [12] found that SPCRC developed more frequently among patients with prior cancer located distally rather than proximally [odds ratio $(\mathrm{OR})=2.30$ ]. Hence, the influence of prior tumor location on the risk of SPCRC needs to be validated by large-scale studies.

In addition, tumor location was reported to influence the survival outcomes of patients with single CRC [14, 15]. Among patients with metastatic CRC, patients with proximal colon cancer had a worse outcome in comparison to those patients with metastatic distal colon cancer or $\mathrm{ReC}$ [16]. Nevertheless, data is lacking concerning the survival rates of CRC survivors suffering from SPCRC. We hypothesize that the survival of CRC patients suffering from SPCRC may differ by the tumor location of the prior CRC.

In the current study, the influence of prior tumor location on SPCRC development and on the survival of SPCRC patients was determined based on the Surveillance, Epidemiology, and End Results (SEER) database. The CRC survivors were divided into three groups according to the prior tumor location, and were designated as the RCC, LCC, and ReC groups. The influence of prior tumor location, as well as latency and age, on the risk of SPCRC was assessed. Whether tumor location of the prior CRC could predict outcomes of the patients with SPCRC was further evaluated.

\section{Methods}

\section{Data source and study cohort}

Cancer incidence was identified from the National Cancer Institute Surveillance, Epidemiology, and End Results (SEER) Program database. The Multiple-Primary session of the SEER"Stat software 8.2.1 was used to estimate the standardized incidence of SPCRC in CRC cases based on the SEER 9 registry data, including individual data from 1973 to 2012. To ensure that recurrences and metastases are not recorded as new primary cancers, SEER registrars adhere to a series of coding rules.

For the present study, index cases included individuals diagnosed with an index colorectal adenocarcinoma confirmed pathologically between 1973 and 2005, in order to allow at least 7 years of follow-up for screening second SPCRC and studying outcomes. Cases that lacked documentation of age at diagnosis or were reported only on death certificates or autopsy reports were excluded. The inclusion criteria for screening SPCRC were as follows: (1) either in situ or malignant; (2) pathologically confirmed; (3) diagnosed subsequent to the index CRC; and (4) diagnosed on or before December 31, 2012. A minimum latency period of 6 months between the diagnoses was required to exclude synchronous cancers. In total, the final study cohort comprised 7290 patients who met the inclusion criteria for SPCRC from the pool of 202,088 index CRC cases.

\section{Estimation of standardized incidence ratio for SPCRC}

To determine the relative risk of SPCRC among all the index CRC cases, the standardized incidence ratio (SIR) was calculated as the ratio of the observed number to the expected number of SPCRCs based on CRC incidence in the general population of the SEER ascertainment areas, with adjustment for sex, age, calendar year, and race. More information on both SEER*Stat software and the method it uses to derive the SIRs are available on the SEER website (available at http:// seer.cancer.gov/resources/). SIRs were calculated for subpopulations with different anatomical sites of the prior tumor, in order to validate if it was reasonable to divide patients into RCC, LCC, and ReC groups according to prior tumor location. Then SIRs for each subgroup were calculated to assess the influence of prior tumor location, latency, and age of prior diagnosis on SPCRC development. A determination of statistical significance was based on a two-sided $p$-value $<0.05$.

\section{Statistical analyses on clinical features}

Detailed information was extracted on sex, ethnicity, stage, grade, age, and year of first diagnosis and anatomical subsites of prior tumors among the cohort. Chi-squared tests were used to assess the differences in demographic and clinical characteristics between the three location groups. The overall survival (OS) was calculated from the date of diagnosis of the prior CRC to the date of all-caused death, or last contact (if the patient was lost to follow-up), or December 31, 2012, whichever occurred first. Regarding cancer-specific survival (CSS), the event is specific to CRC-related death, instead of all-caused death. The survival rate was calculated using the Kaplan-Meier method by SPSS software, version 18.0 (SPSS, Chicago, IL, USA), and was compared using the log-rank test for significant difference by prior tumor location or patient age. Cox regression model was used to assess the independent effect of prior tumor location or age on the hazard ratio (HR) of developing SPCRC after controlling for other variables. All tests were two-tailed, and statistical significance was set at $P<0.05$. 


\section{Results}

\section{Risk of SPCRC}

A total of 7290 cases with prior CRC were shown to develop SPCRC from 1973 to 2005. The overall risk of CRC increased by $27 \%$ (95\% CI: $24 \%-30 \%$ ) in CRC survivors compared to that of the general population (Fig. 1, Additional file 1: Table S1). The risk of SPCRC increased significantly among patients with prior colon cancer (SIRs >1). Furthermore, when grouped by the anatomical sites of the prior tumor, the SIR increased from 1.15 at the cecum to 1.86 at the transverse colon, and then declined from 1.85 at the splenic colon to 1.19 at the rectosigmoid junction. However, the risk of SPCRC in patients with prior $\mathrm{ReC}$ was comparable to the risk of primary CRC in the general population $(\operatorname{SIR}=1)$, and was stable throughout the extended follow-up time.

Among patients with RCC or LCC, the risk of SPCRC increased in the first 60 months after prior diagnosis (Fig. 2a, Additional file 2: Table S2). Moreover, the risk remained elevated by $16 \%-19 \%$ at $60-96$ months among the LCC patients, while the risk was still more than $20 \%$ higher after 108 months' follow-up among the RCC patients, compared to the risk of primary CRC in the general population. The risk of SPCRC decreased significantly with increasing age (Fig. 2b, Additional file 3: Table S3). The tendency was most obvious in the RCC survivors, obvious in the LCC survivors, and then least obvious in the $\mathrm{ReC}$ survivors. The risks were similar to that of the general population in RCC and LCC patients at ages older than 80 years, and in the $\mathrm{ReC}$ patients at ages older than 60 years.

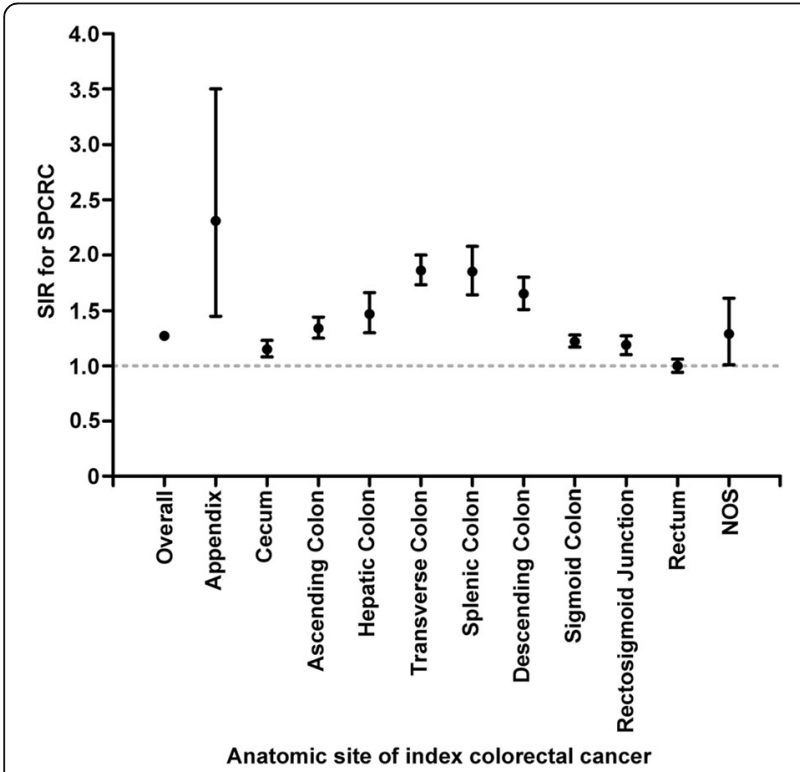

Fig. 1 Standardized incidence ratio for SPCRC by anatomical sites of index colorectal cancer (Abbreviations: SPCRC, subsequent primary colorectal cancer; SIR, standardized incidence ratio; NOS, non-specific)

\section{Patient and tumor characteristics}

Detail information could be retrieved for 6701 patients from the 7290 cases suffering from SPCRC. Comparison was made on clinical features of the initial CRC between the patients with single CRC and patients with SPCRC (Additional file 4: Table S4). Of the 6701 patients with SPCRC, 38.4\% had prior RCC, $47.2 \%$ had prior LCC, and $14.4 \%$ had prior $\mathrm{ReC}$. The median age of these patients at prior diagnosis was 69 years (range, 14-97 years). More than half of them were diagnosed with grade II tumors. The median age was higher for the RCC group (75 years) than for the LCC group (69 years) and Rec group (67 years) (Table 1). Compared to patients with prior LCC and ReC, those patients with prior RCC were more likely to be older, female, and to have more low or undifferentiated grade pathology.

\section{Overall survival by location or age}

The entire cohort had a five-year overall survival (OS) of $73.0 \%$ and a 10 year survival of $48.6 \%$, with a median survival time of 116 months (95\% CI: 112-120). The survival percentages differed according to tumor location of the prior CRC $(P<0.0001)$ (Fig. 3a). The five-year survival percentages were $70.1 \%, 74.2 \%$, and $77.1 \%$ in the RCC, LCC, and $\mathrm{ReC}$ groups, respectively, with corresponding median survival times of 110,116 , and 125 months, respectively. The survival of the cohort also varied according to initial age at diagnosis. The older the patient was when diagnosed with prior CRC, the worse was the outcome $(P<0.0001)$ (Fig. $3 \mathrm{~b})$. The five-year OS percentages were $83.9 \%$ in the $\leq 50$ years of age group, $81.1 \%$ in the $51-60$ years of age group, $79.3 \%$ in the $61-70$ years of age group, $68.5 \%$ in the $71-80$ years of age group, and $49.5 \%$ in the $\geq 81$ years of age group, with corresponding median survival times of $233,170,140,94$, and 60 months, respectively.

\section{Overall survival by both location and age}

In multivariate analyses (Table 2), five factors including stage, race, age, diagnosis year, and sex were indicated as independent prognostic predictors for overall survival, excluding tumor location of the prior CRC. According to stratified analyses (Table 3, Additional file 5: Table S5), the survival difference by prior tumor location within each stratified subgroup disappeared when stratified by the factor of age, but were still significant within location subgroups when stratified by other factors in stratified analyses.

\section{CSS by location or age}

Further comparisons of CSS indicated that the outcomes were similar among the three location groups $(P=0.455)$, but were significantly different among the five age groups $(P<0.0001)$ (Fig. 4, Table 2). Increasing age at diagnosis of 

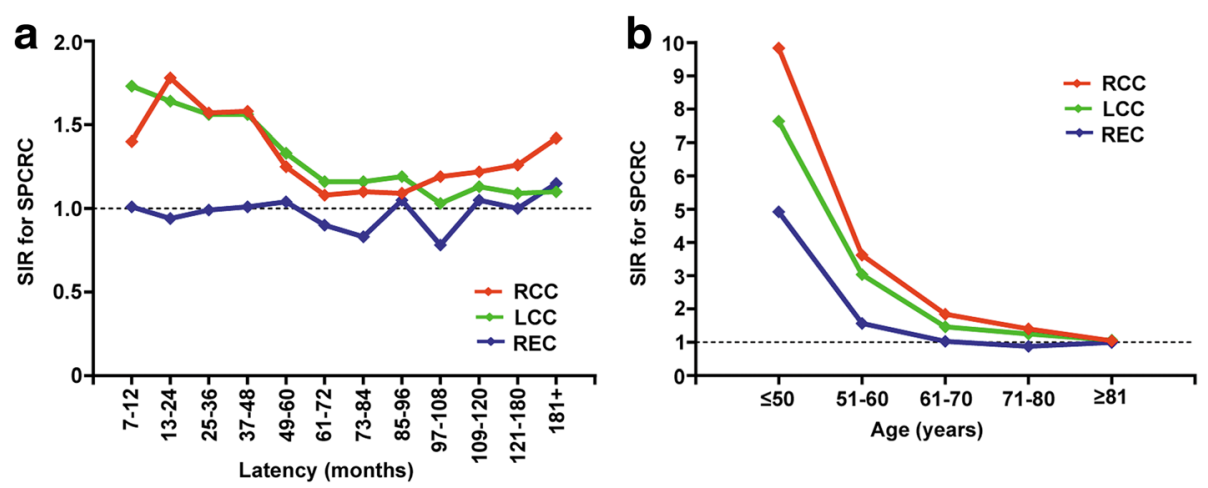

Fig. 2 Standardized incidence ratio for SPCRC by (a) latency or (b) age among colorectal cancer survivors. (Abbreviations: SPCRC, subsequent primary colorectal cancer; SIR, standardized incidence ratio; RCC, right colon cancer; LCC, left colon cancer; ReC, rectal cancer)

Table 1 Characteristics of index colorectal cancer among SPCRC patients according to prior tumor location

\begin{tabular}{|c|c|c|c|c|}
\hline Variable & $\mathrm{RCC}(n=2572)$ & LCC $(n=3166)$ & $\operatorname{ReC}(n=963)$ & $P$ \\
\hline Age at diagnosis & & & & $<0.0001$ \\
\hline median (range) & 70 (14-97) & $68(27-96)$ & $67(22-96)$ & \\
\hline$\leq 50$ & $194(7.5)$ & $209(6.6)$ & $71(7.4)$ & \\
\hline $51-60$ & 339 (13.2) & $568(17.9)$ & $204(21.2)$ & \\
\hline $61-70$ & 776 (30.2) & $1095(34.6)$ & $324(33.6)$ & \\
\hline $71-80$ & $883(34.3)$ & $997(31.5)$ & $270(28.0)$ & \\
\hline$\geq 81$ & $380(14.8)$ & $297(9.4)$ & $94(9.8)$ & \\
\hline Year of diagnosis & & & & 0.158 \\
\hline 1973-1985 & $1273(49.5)$ & $1676(53.0)$ & $485(50.4)$ & \\
\hline 1986-1995 & $886(33.7)$ & $1008(31.8)$ & $332(34.5)$ & \\
\hline 1996-2005 & $433(16.8)$ & $482(15.2)$ & $146(15.1)$ & \\
\hline Race & & & & 0.007 \\
\hline white & $2218(86.2)$ & $2729(86.2)$ & $828(86.0)$ & \\
\hline Black & $214(8.3)$ & $211(6.7)$ & $62(6.4)$ & \\
\hline Others & $140(5.5)$ & $206(7.1)$ & $73(7.6)$ & \\
\hline Gender & & & & $<0.0001$ \\
\hline Female & $1347(52.4)$ & $1389(43.9)$ & $402(41.7)$ & \\
\hline Male & $1225(47.6)$ & $1777(56.1)$ & $561(58.3)$ & \\
\hline Stage & & & & $<0.0001$ \\
\hline Localized & $1008(39.2)$ & $1408(44.4)$ & $554(57.5)$ & \\
\hline Regional & $1395(54.2)$ & $1544(48.8)$ & $338(35.1)$ & \\
\hline Distant & $112(4.4)$ & $132(4.2)$ & $17(1.8)$ & \\
\hline Unknown & $57(2.2)$ & $82(2.6)$ & $54(5.6)$ & \\
\hline Tumor grade & & & & $<0.0001$ \\
\hline Grade I & 307 (11.9) & 438 (13.8) & $123(12.8)$ & \\
\hline Grade II & $1302(50.6)$ & $1797(56.8)$ & $535(55.6)$ & \\
\hline Grade III & $537(20.9)$ & $357(11.3)$ & $116(12.0)$ & \\
\hline Unknown & 426 (16.6) & $574(18.1)$ & 189 (19.6) & \\
\hline
\end{tabular}



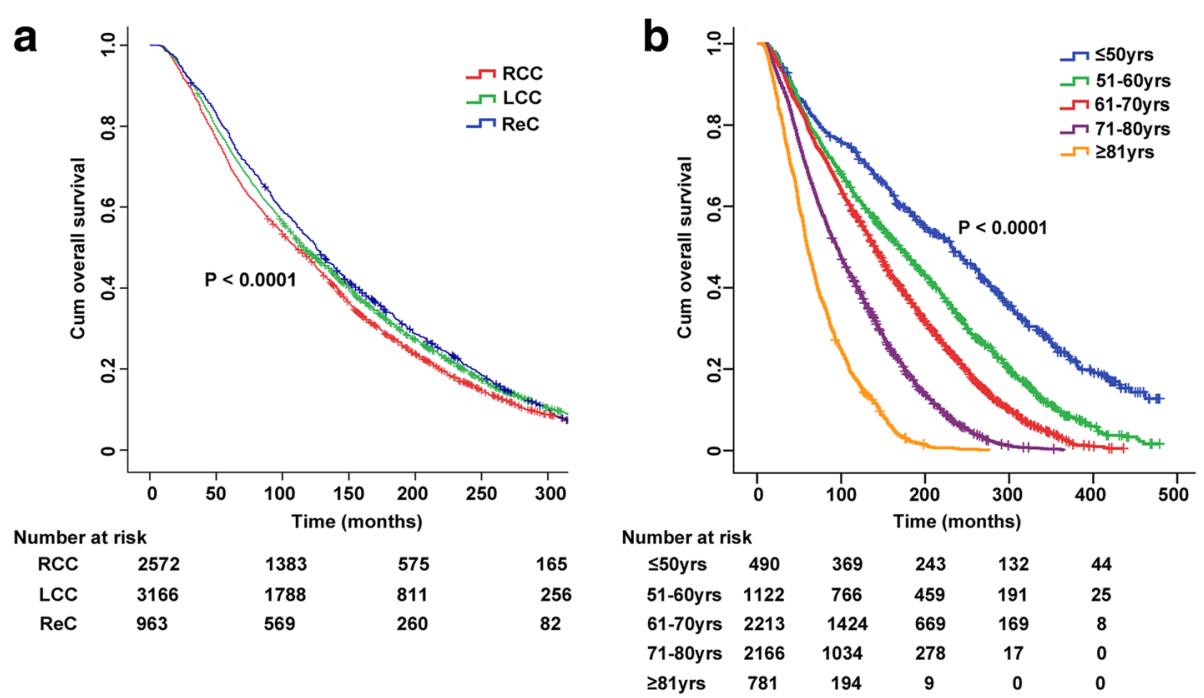

Fig. 3 Overall survival among SPCRC patients by (a) prior tumor location or (b) age at prior diagnosis. (Abbreviations: SPCRC, subsequent primary colorectal cancer; RCC, right colon cancer; LCC, left colon cancer; ReC, rectal cancer)

initial CRC was associated with poorer CSS. However, no obvious difference was found between the 51-60 years of age and the $61-70$ years of age groups $(P=0.579)$.

\section{Discussion}

The current study had two major findings. First, the risk of SPCRC differed by prior tumor location as well as by age and latency. The risk of SPCRC increased both in patients with prior RCC and in patients with prior LCC, whereas, patients with $\mathrm{ReC}$ had similar risks in comparison to that of the general population. The increased risk of SPCRC mainly occurred within the first 5 years after prior diagnosis and among young patients. Second, the survival of patients with SPCRC did not differ significantly by initial CRC locations. Patients with SPCRC after RCC seemed to have a worse OS than those patients with SPCRC after LCC or ReC, but the survival differences by tumor locations disappeared when adjusted or stratified by age. It was age rather than tumor location of prior CRC that was recognized as an independent prognostic factor among patients with SPCRC.

Previous studies have reported that the risk of SPCRC was higher in patients with proximal or distal CRC based on the proportion of SPCRC prevalence $[9,13]$. This study assessed the relative risk of SPCRC using SIR, which was more useful than the simple prevalence. The results indicated that the degree of risk increased and then declined with the cutoff of splenic flexure and with the anatomical sites of prior CRC, located from proximally to distally. This supported the division pattern of colon cancer into right and left subsides. Of significance, all patients with prior colon cancer had an increased risk of SPCRC, with no significant difference observed between the RCC and
LCC groups. In agreement with the current results, a study showed that the risk of SPCRC increased by $40 \%$ among all colon cancer patients, but the risk was similar to that of the general population in $\mathrm{ReC}$ patients [10].

Compared to the RCC and LCC groups, ReC patients were more likely to be younger than 60 years of age and mostly had localized disease. Hence, the ReC survivors may have had a greater chance of being cured, leading to a relatively longer follow-up duration. Furthermore, much more bowel segments were left for the tumor cells to grow among the $\mathrm{ReC}$ survivors after radical surgery, than among those patients with prior colon cancer. However, patients with prior $\mathrm{ReC}$ did not have increased risks, although they seemed to have various advantages in developing SPCRC. ReC may therefore be a distinct form colon cancer.

The risk of SPCRC was obviously concentrated in the first 5 years after prior diagnoses among patients with RCC or LCC. In previous studies, the risk of SPCRC was reported to be mostly focused in the first 3 years after prior diagnoses, because more than half of the SPCRC cases developed during this time period $[17,18]$. We observed that the risks of SPCRC were similar at 3-4 years. In the 5 th year, these patients still had elevated risks of SPCRC, although the degree of risk increase began to decline. Five years later, the risk of SPCRC differed between patients with prior RCC and LCC. Therefore, in the process of SPCRC screening, attention should be given for at least the first 5 years after prior diagnosis among CRC survivors. The location of the prior CRC should also be taken into consideration in individual patients, using a longer surveillance strategy. 
Table 2 Cox proportional hazards analysis of overall survival and cancer-specific survival

\begin{tabular}{|c|c|c|c|c|c|c|c|c|}
\hline \multirow[t]{2}{*}{ Variable } & \multicolumn{4}{|c|}{ Overall survival } & \multicolumn{4}{|c|}{ Cancer-specific survival } \\
\hline & $\mathrm{HR}$ & $95 \% \mathrm{Cl}$ & & $P$ & $\mathrm{HR}$ & $95 \% \mathrm{Cl}$ & & $P$ \\
\hline $\mathrm{RCC}$ & Referent & & & & Referent & & & \\
\hline LCC & 0.94 & 0.89 & 0.99 & 0.028 & 1.01 & 0.93 & 1.10 & 0.811 \\
\hline Rectum & 0.99 & 0.91 & 1.07 & 0.741 & 1.15 & 1.02 & 1.29 & 0.022 \\
\hline Age at diagnosis (year) & & & & $<0.0001$ & & & & $<0.0001$ \\
\hline$\leq 50$ & Referent & & & & Referent & & & \\
\hline $51-60$ & 1.70 & 1.50 & 1.92 & $<0.0001$ & 1.27 & 1.07 & 1.51 & 0.007 \\
\hline $61-70$ & 2.35 & 2.08 & 2.63 & $<0.0001$ & 1.35 & 1.15 & 1.58 & $<0.0001$ \\
\hline $71-80$ & 3.91 & 3.46 & 4.40 & $<0.0001$ & 1.97 & 1.68 & 2.32 & $<0.0001$ \\
\hline$\geq 81$ & 7.18 & 6.26 & 8.22 & $<0.0001$ & 3.23 & 2.68 & 3.91 & $<0.0001$ \\
\hline Stage & & & & $<0.0001$ & & & & $<0.0001$ \\
\hline Localized & Referent & & & & Referent & & & \\
\hline Regional & 1.16 & 1.11 & 1.23 & $<0.0001$ & 1.50 & 1.38 & 1.63 & $<0.0001$ \\
\hline Distant & 2.17 & 1.90 & 2.47 & $<0.0001$ & 3.72 & 3.15 & 4.39 & $<0.0001$ \\
\hline Unknown & 1.21 & 1.04 & 1.40 & 0.014 & 1.48 & 1.19 & 1.85 & 0.001 \\
\hline Grade & & & & 0.949 & & & & 0.491 \\
\hline Grade I & Referent & & & & Referent & & & \\
\hline Grade II & 1.01 & 0.93 & 1.09 & 0.853 & 1.07 & 0.95 & 1.22 & 0.258 \\
\hline Grade III & 1.02 & 0.93 & 1.12 & 0.700 & 1.12 & 0.96 & 1.30 & 0.148 \\
\hline Unknown & 0.98 & 0.98 & 1.07 & 0.673 & 1.10 & 0.95 & 1.27 & 0.198 \\
\hline Race & & & & $<0.0001$ & & & & $<0.0001$ \\
\hline White & Referent & & & & Referent & & & \\
\hline Black & 1.24 & 1.13 & 1.36 & $<0.0001$ & 1.31 & 1.14 & 1.51 & $<0.0001$ \\
\hline Others & 0.94 & 0.85 & 1.04 & 0.223 & 0.84 & 1.80 & 0.99 & 0.038 \\
\hline Gender & & & & $<0.0001$ & & & & $<0.0001$ \\
\hline Male & Referent & & & & Referent & & & \\
\hline Female & 0.74 & 0.70 & 0.78 & $<0.0001$ & 0.83 & 0.77 & 0.90 & $<0.0001$ \\
\hline Year of diagnosis & & & & $<0.0001$ & & & & $<0.0001$ \\
\hline 1973-1985 & Referent & & & & Referent & & & \\
\hline 1986-1995 & 1.27 & 1.20 & 1.36 & $<0.0001$ & 1.24 & 1.13 & 1.36 & $<0.0001$ \\
\hline 1996-2005 & 2.07 & 2.91 & 2.51 & $<0.0001$ & 2.03 & 1.80 & 2.28 & $<0.0001$ \\
\hline
\end{tabular}

Abbreviations: HR Hazard ratio, $\mathrm{Cl}$ confidence interval

Several previous studies showed a higher risk of SPCRC among older patients than among younger patients [19-21]. The majority of the patients with SPCRC were older. This may be attributed to the high prevalence of prior CRC among the elderly patients. The longer follow-up period since prior CRC diagnosis may also have partly contributed to these results. This study showed that when patients were diagnosed with prior colon cancer at a younger age, they had higher risks of SPCRC. The results were consistent with previous studies $[8,22]$. Interestingly, the risk of SPCRC was higher among patients with prior RCC than among those with prior LCC, within each age group. Therefore, the risk of SPCRC should be higher among the RCC patients than among the LCC survivors in the entire cohort. However, no significant difference was observed in the overall risk of SPCRC between these two groups. These results could be due to the different patterns of age distributions between the RCC and LCC groups. Hence, more attention should be paid to young patients with prior colon cancer at the first 5 years after prior diagnosis in clinical surveillance of SPCRC.

One of the major limitations from previous studies was the lack of information on survival outcomes of patients with SPCRC after prior CRC. In our study, a favorable OS was observed among these patients. This may be because these patients underwent a second 
Table 3 Five-year survival rates of SPCRC patients according to prior tumor location in stratified subgroups

\begin{tabular}{|c|c|c|c|c|c|c|c|c|}
\hline \multirow[t]{2}{*}{ Variable } & \multicolumn{4}{|c|}{ Overall survival (\%) } & \multicolumn{4}{|c|}{ Cancer-specific survival (\%) } \\
\hline & $\mathrm{RCC}$ & LCC & $\operatorname{ReC}$ & $P$ & $\mathrm{RCC}$ & LCC & $\operatorname{ReC}$ & $P$ \\
\hline All & 70.1 & 74.2 & 77.1 & $<0.0001$ & 80.9 & 83.7 & 86.7 & 0.455 \\
\hline \multicolumn{9}{|l|}{ Stage } \\
\hline Localized & 76.4 & 79.3 & 82.5 & 0.022 & 89.3 & 88.8 & 91.0 & 0.115 \\
\hline Regional & 67.9 & 72.4 & 70.1 & 0.010 & 77.7 & 81.8 & 81.1 & 0.143 \\
\hline Distant & 36.6 & 45.5 & 32.1 & 0.775 & 46.1 & 52.2 & 51.0 & 0.929 \\
\hline Unknown & 77.2 & 67.1 & 77.8 & 0.046 & 80.5 & 83.2 & 86.7 & 0.436 \\
\hline \multicolumn{9}{|l|}{ Grade } \\
\hline 1 & 72.3 & 80.8 & 78.9 & 0.693 & 86.3 & 90.5 & 87.8 & 0.901 \\
\hline$\|$ & 67.5 & 71.2 & 75.3 & 0.002 & 79.0 & 81.5 & 85.7 & 0.09 \\
\hline III & 67.2 & 73.7 & 74.1 & 0.143 & 78.7 & 82.0 & 81.2 & 0.294 \\
\hline Unknown & 79.8 & 78.9 & 83.1 & 0.361 & 84.3 & 86.2 & 86.2 & 0.545 \\
\hline \multicolumn{9}{|l|}{ Race } \\
\hline Whit & 70.4 & 74.6 & 77.6 & $<0.0001$ & 81.1 & 75.5 & 86.0 & 0.191 \\
\hline Black & 61.7 & 69.2 & 69.4 & 0.687 & 84.1 & 76.9 & 84.4 & 0.150 \\
\hline Others & 75.7 & 73.9 & 78.1 & 0.700 & 87.2 & 79.2 & 87.0 & 0.575 \\
\hline \multicolumn{9}{|l|}{ Gender } \\
\hline Male & 67.1 & 72.2 & 73.8 & 0.011 & 79.4 & 83.0 & 73.8 & 0.452 \\
\hline Female & 72.8 & 76.8 & 81.8 & 0.001 & 82.3 & 84.6 & 88.9 & 0.454 \\
\hline \multicolumn{9}{|c|}{ Year of diagnosis } \\
\hline 1973-1985 & 77.1 & 80.2 & 84.3 & 0.002 & 85.1 & 87.5 & 90.6 & 0.372 \\
\hline 1986-1995 & 71.2 & 74.0 & 76.5 & 0.768 & 83.0 & 83.6 & 85.1 & 0.514 \\
\hline 1996-2005 & 47.1 & 53.9 & 54.8 & 0.327 & 63.1 & 69.4 & 75.7 & 0.870 \\
\hline \multicolumn{9}{|c|}{ Age at diagnosis } \\
\hline$\leq 50$ & 83.5 & 81.3 & 90.1 & 0.779 & 87.5 & 83.1 & 91.5 & 0.053 \\
\hline $51-60$ & 76.1 & 80.8 & 87.7 & 0.295 & 82.8 & 86.0 & 92.5 & 0.344 \\
\hline $61-70$ & 77.6 & 80.1 & 80.9 & 0.427 & 85.5 & 87.4 & 89.6 & 0.965 \\
\hline $71-80$ & 67.6 & 68.5 & 71.5 & 0.458 & 80.4 & 81.3 & 83.6 & 0.452 \\
\hline$\geq 81$ & 48.2 & 52.2 & 46.8 & 0.225 & 67.4 & 72.0 & 65.8 & 0.470 \\
\hline
\end{tabular}

Abbreviations: SPCRC Subsequent primary colorectal cancer, RCC Right colon cancer, LCC Left colon cancer, ReC Rectal cancer

screening by the inclusion criteria of SPCRC development. Patients with early stage disease and long follow-up times had a greater possibility of developing SPCRC. In further stratified analyses by prior tumor location, outcomes were poorer in the RCC group than in the LCC or ReC groups, but were similar between the latter two groups. These comparable results between any two of the three groups were in agreement with the outcomes among patients with single CRC $[14,15]$. To some extent, it could be speculated that the occurrence of SPCRC does not change the survival pattern of CRC patients. Hildebrand et al. also reported that no significant difference was detected in survival times between patients with and without secondary or multiple primary tumors [23]. They further showed that the presence of SPCRC was not considered as an independent prognostic factor in patients with a prior CRC, as assessed on the basis of direct survival comparisons. Multiple primary tumors are not necessarily associated with a worse outcome, therefore patients should receive curative intent surgery and appropriate follow-up care.

The influence of age on the survival of patients with single CRC has been studied, although the results are not consistent [24-28]. Previous studies have not focused on the impact of age on the outcomes in patients with SPCRC after CRC. The current study identified age as a significant prognostic predictor among SPCRC patients with prior CRC. A younger age was associated with a favorable outcome. In addition to their relatively longer life expectancy, the distinct tumor behavior may also play a role in the better survival of the younger patients, when compared to the older patients. Young patients with multiple primary CRCs usually had a positive family history and were more likely to carry germline mutations, such as the deficient mismatch repair gene [29]. Screening using more accurate biomarkers should help to identify these patients. For this purpose, an intensive biomarker study could be conducted in the future.

Among the patients with SPCRC, the tumor location of prior CRC was not an independent overall prognostic factor. The overall survival difference by tumor location disappeared only when adjusted or stratified by the factor of age. Therefore, the survival difference by tumor location mainly resulted from the special pattern of age distribution in the three location groups. The highest proportion of the patients $\geq 70$ years of age, and the association between older age and unfavorable outcomes, led to the worst outcomes in the RCC survivors. In addition, the CSS percentages were similar among the three location groups, but were different among the five age groups. These results indicated that tumor behavior might vary substantially according to age of prior CRC diagnosis, rather than according to prior tumor location. In the clinical surveillance of CRC survivors, age is therefore a more important factor than tumor location of the prior CRC. Young patients with prior CRC had a higher risk for SPCRC, but a better survival than that of the older patients, because they received standard and curable treatment and had longer follow-up times.

In this retrospective study, a large sample size was reliably identified from the SEER database. All patients received at least a 7 year follow-up until their death, or to the deadline of December, 2012. In our study, the survival of patients with SPCRC after prior CRC was evaluated.

This study had some limitations. First, in some cases, data were lacking concerning the detailed records of patient follow-ups, and concerning the specific advice given to patients about their surveillance methods. Second, data on genetic variations were not included, 

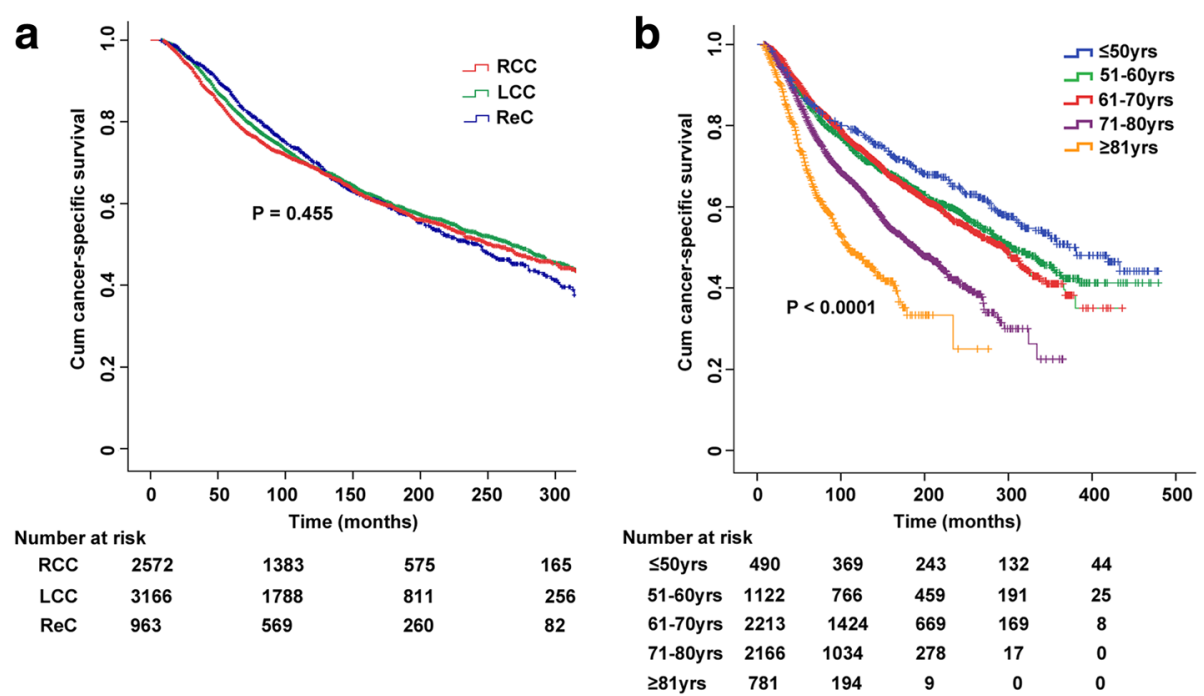

Fig. 4 Cancer-specific survival among SPCRC patients by (a) prior tumor location or (b) age at prior diagnosis. (Abbreviations: SPCRC, subsequent primary colorectal cancer; RCC, right colon cancer; LCC, left colon cancer; ReC, rectal cancer)

making it impossible to directly compare our findings with molecular studies. In the future, more detailed genetic variation studies will be required to identify patients at high risk in order to include a better individual clinical surveillance.

\section{Conclusions}

The risk of SPCRC increased among patients with both prior RCC and LCC, but not among those patients with $\mathrm{ReC}$. The differences in survival outcomes in CRC survivors suffering from SPCRC were largely correlated with patient age, but not with prior tumor location. In the clinical surveillance of CRC survivors, age at prior diagnosis is a more important factor than prior CRC location.

\section{Additional files}

Additional file 1: Table S1. Standardized incidence ratio for SPCRC by anatomical sites of index colorectal cancer. (DOCX $27 \mathrm{~kb}$ )

Additional file 2: Table S2. Standardized incidence ratio for SPCRC by latency among colorectal cancer survivors. (DOCX $29 \mathrm{~kb}$ )

Additional file 3: Table S3 Standardized incidence ratio for SPCRC by age among colorectal cancer survivors. (DOCX $26 \mathrm{~kb}$ )

Additional file 4: Table S4. Characteristics of index cancer among patients with single colorectal cancer and patients with SPCRC. (DOCX $25 \mathrm{~kb}$ )

Additional file 5:Table S5. Median survival of SPCRC patients according to prior tumor location in stratified subgroups. (DOCX $35 \mathrm{~kb}$ )

\section{Abbreviations}

Cl: confidence interval; CRC: colorectal cancer; CSS: cancer-specific survival; HR: hazard ratio; LCC: left colon cancer; OR: Odds ratio; OS: overall survival; RCC: right colon cancer; ReC: rectal cancer; SEER: Surveillance, Epidemiology, and End Results Program database; SIR: standardized incidence ratio; SPCRC: subsequent primary colorectal cancer

\section{Acknowledgments}

The authors acknowledge the efforts of the SEER Program tumor registries in the creation of the SEER database. The authors also thank the editor and reviewers for careful review and insightful comments, which have led to a significant improvement of the manuscript.

Funding

No funding was obtained for this study.

Availability of data and materials

All analyzed data are publicly available at the SEER website (http:// www.seer.cancer.gov), and should be requested under the approval of the SEER Program administration.

\section{Authors' contributions}

JiY, XD, JinY carried out conception, study design and data interpretation. JiY wrote the original draft. SL, YW, ML, DD, LZ and ZC carried out data analysis, check and editing. BW, FW and YS carried out visualization and editing. EL, MY and JinY carried out validation, writing-review and editing. MY guided the methodology. JinY carried out supervision. All authors read and approved the final manuscript.

Ethics approval and consent to participate

The study was approved by the Ethics Committee of the First Affiliated Hospital of Xi'an Jiaotong University. Since it did not include interaction with humans or use personal identifying information, the informed consent was not required for this research. We were permitted to have Internet access after our signed data-use agreement (http//seer.cancer.gov/data/sample-dua.html) was approved by the SEER administration (Reference number: 10,803-Nov2015).

\section{Consent for publication}

Not applicable.

\section{Competing interests}

The authors declare that they have no competing interest.

\section{Publisher's Note}

Springer Nature remains neutral with regard to jurisdictional claims in published maps and institutional affiliations.

\section{Author details}

'Departments of Medical Oncology, The First Affiliated Hospital of Xi'an Jiaotong University, 277 Yanta West Road, Xi'an 710061, Shaanxi Province, People's Republic of China. ${ }^{2}$ Division of Epidemiology \& Disease Control, 
School of Public Health, The University of Texas Health Science Center at Houston, Houston, TX, USA. ${ }^{3}$ Breast Surgical Oncology, The University of Texas MD Anderson Cancer Center, Houston, TX, USA.

Received: 19 August 2016 Accepted: 8 November 2017

Published online: 22 November 2017

\section{References}

1. Ikeda Y, Kishihara F, Saitsu H, Saku M, Maehara Y. Second primary cancer after surgery for colorectal cancer without lymph node metastasis. HepatoGastroenterology. 2007:54:1962-5.

2. Dasgupta P, Youlden DR, Baade PD. Multiple primary cancers among colorectal cancer survivors in Queensland, Australia, 1996-2007. Cancer Causes Control. 2012;23:1387-98.

3. Balleste B, Bessa X, Pinol V, Castellvi-Bel S, Castells A, Alenda C, et al. Detection of metachronous neoplasms in colorectal cancer patients: identification of risk factors. Dis Colon Rectum. 2007;50:971-80.

4. Newton KF, Green K, Walsh S, Lalloo F, Hill J, Evans DG. Metachronous colorectal cancer risk in patients with a moderate family history. Colorectal Dis. 2013:15:309-16

5. Cirillo L, Urso ED, Parrinello G, Pucciarelli S, Moneghini D, Agostini M, et al. High risk of rectal cancer and of metachronous colorectal cancer in probands of families fulfilling the Amsterdam criteria. Ann Surg. 2013;257:900-4.

6. Das A, Chak A, Cooper GS. Temporal trend in relative risk of second primary colorectal cancer. Am J Gastroenterol. 2006;101:1342-7.

7. Shureiqi I, Cooksley CD, Morris J, Soliman AS, Levin B, Lippman SM. Effect of age on risk of second primary colorectal cancer. J Natl Cancer Inst. 2001; 93(16):1264-6.

8. Levi F, Randimbison L, Blanc-Moya R, Maspoli-Conconi M, Rosato V, Bosetti C, et al. High constant incidence of second primary colorectal cancer. Int J Cancer. 2013;132:1679-82.

9. Gervaz P, Bucher P, Neyroud-Caspar I, Soravia C, Morel P. Proximal location of colon cancer is a risk factor for development of metachronous colorectal cancer: a population-based study. Dis Colon Rectum. 2005;48:227-32.

10. Raj KP, Taylor TH, Wray C, Stamos MJ, Zell JA. Risk of second primary colorectal cancer among colorectal cancer cases: a population-based analysis. J Carcinog. 2011:10:6.

11. Rennert G, Robinson E, Rennert HS, Neugut Al. Clinical characteristics of metachronous colorectal tumors. Int J Cancer. 1995:60:743-7.

12. Borda A, Martinez-Penuela JM, Borda F, Munoz-Navas M, Jimenez FJ, Carretero C. Drawing up an individual risk index for development of metachronous neoplastic lesions in resected colorectal cancer. Rev Esp Enferm Dig. 2012;104:291-7.

13. Lan YT, Lin JK, Li AF, Lin TC, Chen WS, Jiang JK, et al. Metachronous colorectal cancer: necessity of post-operative colonoscopic surveillance. Int $J$ Color Dis. 2005:20:121-5.

14. Price TJ, Beeke C, Ullah S, Padbury R, Maddern G, Roder D, et al. Does the primary site of colorectal cancer impact outcomes for patients with metastatic disease? Cancer. 2015;121:830-5.

15. Loupakis F, Moretto R, Aprile G, Muntoni M, Cremolini C, lacono D, et al. Clinico-pathological nomogram for predicting BRAF mutational status of metastatic colorectal cancer. Br J Cancer. 2016;114:30-6.

16. Wu CC, Hsu TW, Chang CM, Yu CH, Lee CC. Age-adjusted Charlson comorbidity index scores as predictor of survival in colorectal cancer patients who underwent surgical resection and chemoradiation. Medicine. 2015;94:e431.

17. Hollington $P$, Tiong $L$, Young G. Timing and detection of metachronous colorectal cancer. ANZ J Surg. 2011;81:272-4.

18. Chen TA, Horng JT, Lin WC. Metachronous colorectal cancer in Taiwan: analyzing 20 years of data from Taiwan cancer registry. Int J Clin Oncol. 2013;18:267-72

19. Lee SY, Kim BC, Han KS, Hong CW, Sohn DK, Park SC, et al. Incidence and risk factors of metachronous colorectal neoplasm after curative resection of colorectal cancer in Korean patients. J Dig Dis. 2014;15:367-76.

20. Moon CM, Cheon JH, Choi EH, Kim ES, Park JJ, Han SY, et al. Advanced synchronous adenoma but not simple adenoma predicts the future development of metachronous neoplasia in patients with resected colorectal cancer. J Clin Gastroenterol. 2010;44:495-501.

21. Nah BK, Kim SM, Lee YS, Yang HW, Seo SW, Sung JK, et al. Patterns of metachronous adenoma after colorectal cancer surgery. Korean J Gastroenterol. 2004:44:212-6.
22. Liang YH, Shao YY, Chen HM, Lai CL, Lin ZZ, Kuo RN, et al. Young patients with colorectal cancer have increased risk of second primary cancers. Jpn J Clin Oncol. 2015;45:1029-35.

23. Hildebrand P, Humke J, Oevermann E, Schloericke E, Burk C, Roblick UJ, et al. Influence of second or multiple tumours on the prognosis of patients with colorectal cancer. Acta Chirurgica lugoslavica. 2012;59:31-8.

24. Abdelsattar ZM, Wong SL, Regenbogen SE, Jomaa DM, Hardiman KM, Hendren S. Colorectal cancer outcomes and treatment patterns in patients too young for average-risk screening. Cancer. 2016;122(6):929-34.

25. McKay A, Donaleshen J, Helewa RM, Park J, Wirtzfeld D, Hochman D, et al. Does young age influence the prognosis of colorectal cancer: a populationbased analysis. World J Surg Oncol. 2014;12:370.

26. Fu J, Yang J, Tan $Y$, Jiang $M$, Wen $F$, Huang $Y$, et al. Young patients $(</=35$ years old) with colorectal cancer have worse outcomes due to more advanced disease: a 30-year retrospective review. Medicine. 2014;93:e135.

27. Chan KK, Dassanayake B, Deen R, Wickramarachchi RE, Kumarage SK, Samita S, et al. Young patients with colorectal cancer have poor survival in the first twenty months after operation and predictable survival in the medium and long-term: analysis of survival and prognostic markers. World J Surg Oncol. 2010:8:82.

28. Murata A, Akiyoshi T, Ueno M, Fukunaga Y, Nagayama S, Fujimoto $Y$, et al. Clinicopathological characteristics of young patients with sporadic colorectal cancer. Surg Today. 2016;46(10):1166-75.

29. Seppälä T, Pylvänäinen K, Renkonen-Sinisalo L, Böhm J, Kuopio T, Järvinen HJ, et al. Diagnosis and treatment of lynch syndrome. Duodecim. 2016; 132(3):233-40

\section{Submit your next manuscript to BioMed Central and we will help you at every step:}

- We accept pre-submission inquiries

- Our selector tool helps you to find the most relevant journal

- We provide round the clock customer support

- Convenient online submission

- Thorough peer review

- Inclusion in PubMed and all major indexing services

- Maximum visibility for your research

Submit your manuscript at www.biomedcentral.com/submit
) Biomed Central 